\title{
Bioéthique dans le monde
}

\section{Jean Martin}

Dr med., membre de la rédaction

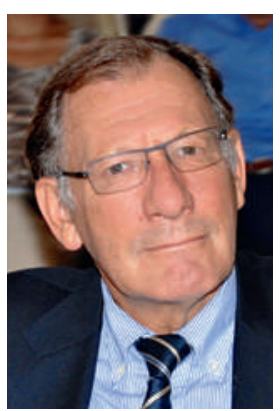

Je reçois en ligne le bulletin hebdomadaire BioEdge (www.bioedge.org), dont je parcours à chaque fois avec intérêt les informations dans le vaste domaine de la bioéthique. Son responsable est un journaliste scientifique basé à Sydney, Michael Cook, qui anime un second site stimulant, Mercatornet (www.mercatornet.com), dont le logo est «Navigating modern complexities».

Les articles de BioEdge touchent un éventail d'enjeux. A propos d'un défi majeur, Michael Cook commente l'encyclique Laudato si du Pape François: «En un sens, Laudato si est une méditation large sur la bioéthique. Il y a toujours eu une tension entre une 'bioéthique de la liberté', qui met l'accent sur l'autonomie de la personne et la protection de son intégrité, et une 'bioéthique globale', qui inclut l'activité humaine dans le milieu.» C'est dans ce sens que François parle d'écologie intégrale.

Bien que souhaitant développer une perspective mondiale, BioEdge parle plus d'Europe, d'Amérique du Nord et du Pacifique Sud - mais le fait est que beaucoup de travaux bioéthiques sont issus de ces régions. Les thèmes de l'euthanasie et du suicide assisté, d'actualité dans plusieurs pays, sont souvent traités. En septembre 2015, est discuté l'avis de Richard Horton, rédacteur du Lancet, qui observe un consensus croissant sur l'acceptabilité de l'assistance au suicide. Ceci au moment où le Parlement britannique refuse à 3 contre 1 une modification légale dans ce sens et où, à l'inverse, le Gouverneur de Californie signe une loi légalisant cette assistance dans son Etat.

Au moment où ces lignes sont écrites, BioEdge présente une prise de position du Comité international de bioéthique (CIB) de l'UNESCO: «La thérapie génique représente une ligne de partage en médecine. Le genome editing (sélection/aménagement d'un génome) est une entreprise prometteuse. Toutefois, des interventions sur le génome ne sont acceptables que pour des raisons préventives, diagnostiques ou thérapeutiques et sans que des modifications puissent être transmises aux descendants.» Le CIB demande un large débat sur le potentiel y relatif de la science et, à ce stade, un moratoire sur le genome editing. Présentation d'un travail récent du bioéthicien hollando-américain Henk ten Have, pour qui la responsabilité éthique a son origine dans la réalité de la vulnérabilité humaine: "Confronté à la mondialisation et aux défis de la pauvreté, des inégalités, de la dégradation environnementale, le discours bioéthique contemporain ne suffit plus. Il doit être augmenté par un plus large éventail de principes allant au-delà de la perspective individuelle et incluant une attention à la vulnérabilité, élément fondamental de notre nature [...] L'éthique ne rendra pas justice à sa mission si elle fait abstraction de la dimension sociale, et si elle néglige les effets délétères que les mécanismes du marché ont sur la vie de la société.»

Parmi les multiples problématiques abordées: la question (pérenne) de l'objection de conscience, dans divers domaines, la PMA et ses développements parfois ébouriffants, des questions liées à la transplantation, le harcèlement sexiste des chirurgiens femmes en Australie... Une citation du 27 septembre susceptible d'amuser: "Le but des transhumanistes est l'extension indéfinie de la vie. Mais, selon Steve Fuller, un leader dans le domaine, sociologue de l'Université de Warwick, c'est une bataille difficile de convaincre un public stupide, qui accepte passivement le futur misérable qui est actuellement le nôtre»!

La pondération, la pesée attentive des enjeux, est une dimension majeure en éthique.

En cherchant ce qui concerne la Suisse: en août 2015, un article sur l'arrêt du Tribunal fédéral refusant à un couple gay la paternité d'un enfant né par mère porteuse à l'étranger. En mai était présenté l'enjeu électoral du 14 juin (modification constitutionnelle autorisant le DPI). Notre pays est aussi présent à propos d'assistance au suicide: en 2104, la loi neuchâteloise enjoignant les EMS d'accepter de telles assistances; auparavant avait été mentionné dans le même canton l'acquittement d'un médecin mis en cause dans un cas de suicide assisté.

Il n'est pas rare d'être déçu de la qualité des articles lus dans les médias sur des sujets qu'on connaît!... Je ne saurais me prononcer sur l'ensemble des livraisons de BioEdge mais il me paraît que ses textes et commentaires, ni ultra-libéraux ni indûment conservateurs, sont généralement bien informés et équilibrés. Et la pondération, la pesée attentive des enjeux, est une dimension majeure en éthique. 